\title{
Optimización del muestreo de invertebrados tropicales: Un ejemplo con escarabajos coprófagos (Coleoptera: Scarabaeinae) en Venezuela
}

\author{
José Rafael Ferrer-Paris ${ }^{1}$, Ada Sánchez-Mercado ${ }^{1} \&$ Jon Paul Rodríguez ${ }^{2}$ \\ 1. Laboratorio de Ecología Espacial, Centro de Estudios Botánicos y Agroforestales, Instituto Venezolano de \\ Investigaciones Científicas, Apartado 20632, Caracas, 1020-A, Venezuela; asanchez@ivic.gob.ve \\ 2. Centro de Ecología, Instituto Venezolano de Investigaciones Científicas; jonpaul@ivic.gob.ve
}

\author{
Recibido 16-I-2012. Corregido 05-VI-2012. Aceptado 03-VII-2012.
}

\begin{abstract}
Sampling optimization for tropical invertebrates: An example using dung beetles (Coleoptera: Scarabaeinae) in Venezuela. The development of efficient sampling protocols is an essential prerequisite to evaluate and identify priority conservation areas. There are few protocols for fauna inventory and monitoring in wide geographical scales for the tropics, where the complexity of communities and high biodiversity levels, make the implementation of efficient protocols more difficult. We proposed here a simple strategy to optimize the capture of dung beetles, applied to sampling with baited traps and generalizable to other sampling methods. We analyzed data from eight transects sampled between 2006-2008 with the aim to develop an uniform sampling design, that allows to confidently estimate species richness, abundance and composition at wide geographical scales. We examined four characteristics of any sampling design that affect the effectiveness of the sampling effort: the number of traps, sampling duration, type and proportion of bait, and spatial arrangement of the traps along transects. We used species accumulation curves, rank-abundance plots, indicator species analysis, and multivariate correlograms. We captured 40337 individuals (115 species/morphospecies of 23 genera). Most species were attracted by both dung and carrion, but two thirds had greater relative abundance in traps baited with human dung. Different aspects of the sampling design influenced each diversity attribute in different ways. To obtain reliable richness estimates, the number of traps was the most important aspect. Accurate abundance estimates were obtained when the sampling period was increased, while the spatial arrangement of traps was determinant to capture the species composition pattern. An optimum sampling strategy for accurate estimates of richness, abundance and diversity should: (1) set 50-70 traps to maximize the number of species detected, (2) get samples during 48-72 hours and set trap groups along the transect to reliably estimate species abundance, (3) set traps in groups of at least 10 traps to suitably record the local species composition, and (4) separate trap groups by a distance greater than $5-10 \mathrm{~km}$ to avoid spatial autocorrelation. For the evaluation of other sampling protocols we recommend to, first, identify the elements of sampling design that could affect the sampled effort (the number of traps, sampling duration, type and proportion of bait) and their spatial distribution (spatial arrangement of the traps) and then, to evaluate how they affect richness, abundance and species composition estimates. Rev. Biol. Trop. 61 (1): 89-110. Epub 2013 March 01.
\end{abstract}

Key words: biodiversity inventories, NeoMaps, Scarabaeidae, species composition, species richness, systematic sampling.

Ante la crisis global de pérdida de biodiversidad, una de las principales acciones de conservación ha sido la identificación de áreas prioritarias para la protección de las características naturales de los ecosistemas (Margules \& Pressey 2000). Para ello es necesario disponer de datos sobre los patrones de distribución y abundancia de especies que permitan detectar diferencias espaciales en la riqueza y diversidad de especies, y que consideren su grado de endemismo, representatividad y complementariedad (Colwell 2009).

Generar datos sobre distribución y abundancia que sean útiles para la toma de decisiones 
en conservación y manejo requiere del desarrollo de inventarios que apliquen protocolos de muestreo rigurosos, factibles, eficientes y sistemáticos (Cardoso et al. 2009). Existen varios ejemplos de protocolos de observación y detección de vertebrados e invertebrados en zonas templadas o subtropicales que han sido aplicados exitosamente en escalas geográficas amplias, como parte de programas de inventario y monitoreo (Ward \& Lariviere 2004, Carr III \& de Stoll 2005, Schieck \& Stelfox 2006, BBS 2010, Watson 2010). Sin embargo, existen pocos ejemplos en regiones tropicales (Villarreal et al. 2004, Noriega \& Fagua 2009, Ahumada et al. 2011) y particularmente para invertebrados, resulta difícil implementar protocolos de muestreo efectivos dada la gran variabilidad en la probabilidad de detección de los individuos (Fisher 1999, Walpole \& Sheldon 1999, Ward \& Lariviere 2004, Cardoso et al. 2008, Gardner et al. 2008, Radtke et al. 2010).

Usualmente, los inventarios de invertebrados se enfocan en un grupo taxonómico indicador y consisten en hacer muestreos localizados con uno o varios métodos de captura a lo largo de transectos ubicados en varios tipos de hábitat dentro de una zona de interés reducida (Colwell \& Coddington 1994). El objetivo de estos inventarios locales es obtener una estimación de riqueza, abundancia o composición en un determinado tipo de hábitat, para luego ser comparados con los estimadores obtenidos en otro tipo de hábitat (Larsen \& Forsyth 2005). No obstante, si el objetivo del inventario es monitorear tendencias espaciales o temporales y discernir si los cambios locales se enmarcan dentro de tendencias, regionales o nacionales, se necesita cubrir una gama amplia de escalas geográficas. Para lograr esto se requiere de un diseño muestreal eficiente, que permita extender y estandarizar los muestreos locales y replicarlos adecuadamente en diferentes regiones con características ambientales heterogéneas. Un diseño muestreal robusto permitirá a su vez obtener estimadores confiables de riqueza, diversidad y composición de especies (Buzas \& Hayek 1996, Gotelli \& Colwell 2001).
Un diseño muestreal robusto debe tomar en cuenta tanto los factores que influyen directamente sobre las variables de interés (biogeografía, heterogeneidad de hábitats, estructura del paisaje, condiciones climáticas, entre otros, Pierre et al. 2008), así como los que afectan la detección de las especies y por tanto, influyen sobre la confiabilidad de los estimadores (especialmente el esfuerzo de muestreo, la efectividad de los métodos de captura y la heterogeneidad espacial; Kéry \& Schmid 2004). En este trabajo examinamos los resultados de varios muestreos preliminares de la Iniciativa para el Mapeo de la Biodiversidad Neotropical (NeoMapas, Rodríguez \& Sharpe 2002) aplicados a escarabajos coprófagos (Coleoptera, Scarabaeinae), con el fin de proponer un diseño de muestreo estandarizado que permita obtener estimadores confiables de riqueza, composición y abundancia en distintas regiones a nivel nacional. Los escarabajos coprófagos son uno de los grupos de artrópodos más utilizados en el estudio de la biodiversidad neotropical (Halffter \& Favila 1997) y son de gran interés para programas de inventario y monitoreo por su alta diversidad y abundancia en la región, con cerca de 1300 especies y 70 géneros (Vaz-deMello et al. 2011), así como por su importante papel ecológico, ya que son un componente clave en el reciclaje de nutrientes y dispersión de semillas al alimentarse principalmente de excrementos y de materia en descomposición (Halffter \& Favila 1997, Spector 2006, Nichols et al. 2008).

El método más utilizado para el muestreo de escarabajos coprófagos son las trampas de caída cebadas, las cuales utilizan la afinidad diferencial de las especies por determinados tipos de cebos para atraer una gran variedad de especies de escarabajos (Halffter \& Favila 1997). Un diseño muestreal óptimo para escarabajos coprófagos representa un compromiso entre el grado de independencia entre las trampas, el tiempo y el costo asociado con el arreglo espacial de los grupos de trampas y la eficiencia en estratificar las unidades de muestreo entre los distintos tipos de hábitats (Rohr et al. 2007). En este sentido, los 
aspectos más relevantes del diseño muestreal a tomar en cuenta son: (i) el número de trampas, (ii) la duración del muestreo, (iii) el arreglo espacial de las trampas y (iv) el tipo de cebo utilizado (Villarreal et al. 2004, Filgueiras et al. 2009, Flechtmann et al. 2009, Noriega \& Fagua 2009). Específicamente, nuestro objetivo consistió en evaluar el efecto de estos cuatro aspectos del diseño muestreal en los estimadores de riqueza, composición y abundancia a dos escalas diferentes. Primero, a una escala local (dentro de un transecto), a fin de detectar y cuantificar los cambios en la biodiversidad a través del tiempo. Segundo, a una escala regional, al comparar dos o más transectos para detectar cambios tanto en riqueza de especies, como de composición.

\section{MATERIALES Y MÉTODOS}

Muestreo de escarabajos coprófagos: Los muestreos analizados en este estudio fueron realizados durante 2005, 2006 y 2008 en ocho regiones de Venezuela y forman parte de un estudio piloto dentro de la Iniciativa para el Mapeo de la Biodiversidad Neotropical (NeoMapas, Rodríguez \& Sharpe 2002; Cuadro 1).

En cada localidad, se ubicó un transecto a lo largo de una carretera secundaria cubriendo la gama más amplia posible de altitudes y tipos de vegetación. Seis de los transectos tuvieron $40 \mathrm{~km}$ de longitud (NM05, NM08, NM09, NM24, NM26 y NM93), mientras que los otros dos (NM92 y NM97) fueron transectos con dimensiones reducidas $(<10 \mathrm{~km})$. En general, el protocolo consistió en ubicar un número variable de grupos de trampas de caída cebadas con $\sim 20 \mathrm{~g}$ de heces humanas o carroña en descomposición, con una distancia mínima de $\sim 50 \mathrm{~m}$ entre trampas (Cuadro 1, Fig. 1). En todos los casos, la unidad de muestreo fue la trampa de caída (Larsen \& Forsyth 2005) y se cuantificó el esfuerzo de muestreo como la suma de las horas que cada trampa se mantuvo activa (horas/trampa). Dado que el número de individuos capturados por las trampas de caídas es normalmente bajo, se ha sugerido que los inventarios que utilizan este método de captura podrían hacerse más efectivos si se incrementa el esfuerzo de muestreo (JiménezValverde \& Lobo 2005). No obstante, siguiendo la recomendación de Vennila \& Rajagopal (1999), variamos dentro de un mismo transecto el tamaño de los grupos de trampas con el fin de muestrear un mayor número de microhábitats y evaluar el efecto de este factor sobre los estimadores de riqueza. Así, colocamos grupos grandes de trampas (entre 11-20) en el transecto NM97, grupos medianos (entre 6-10 trampas) en los transectos NM05, NM93 y NM92 y grupos pequeños (1-5 trampas) en NM24, NM26 y NM09. Adicionalmente, también variamos en cada localidad de muestreo los tipos de cebos utilizados y la distancia entre grupos de trampas a lo largo del transecto.

Las trampas de caída fueron construidas con envases plásticos de $12.5 \mathrm{~cm}$ de diámetro y $13 \mathrm{~cm}$ de profundidad $\left(420 \mathrm{~cm}^{3}\right)$, cubiertos por un plato plástico para proteger la trampa del sol y la lluvia (Spector 2006). Las trampas contuvieron una solución preservante constituida por agua y etanol técnico al 75\% (proporciones $40 \%$ y $60 \%$ respectivamente). El cebo fue colocado en una bolsa de tul de nylon y luego suspendido por encima del líquido preservante utilizando un alambre enterrado en el suelo. El material fue recolectado con una frecuencia diaria o interdiaria por cuatro días consecutivos (tiempos de revisión 24, 48, 72 y 96 horas). Por motivos logísticos, no se realizaron sustituciones de cebo durante todo el tiempo de muestreo, y se recogieron las trampas cuando el cebo fue removido por algún animal. Adicionalmente, se tomó una descripción detallada del tipo de hábitat, así como de las principales perturbaciones (e.g. quemas, deforestación, construcciones de viviendas, entre otras). Sin embargo, esta información no fue analizada en el presente trabajo y se espera utilizarla en estudios futuros sobre cambios espaciales en la composición y riqueza de especies.

El material recolectado se limpió en cada revisión pasando la muestra por un colador y enjuagando con solución preservante limpia. Seguidamente, fue transferido a bolsas plásticas con alcohol técnico/solución preservante 


$$
\begin{aligned}
& \| m=\ldots
\end{aligned}
$$

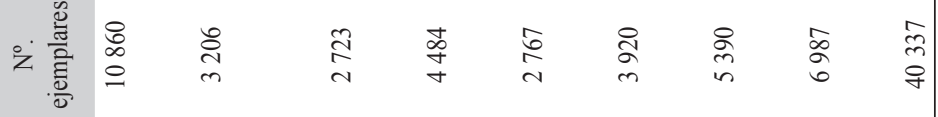

$$
\begin{aligned}
& \text { 安总 }
\end{aligned}
$$

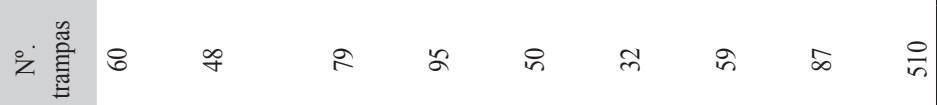

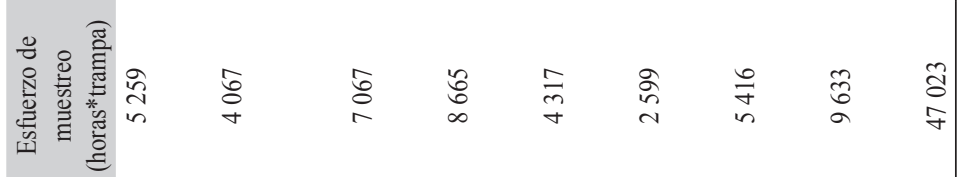

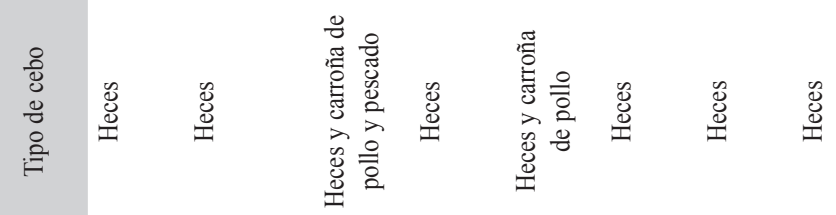

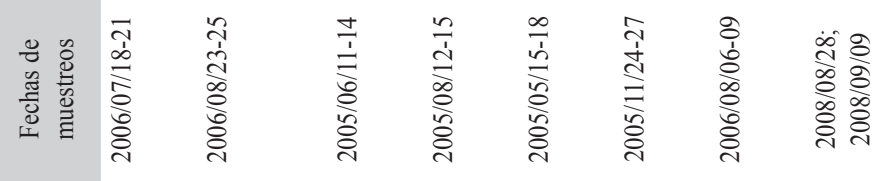

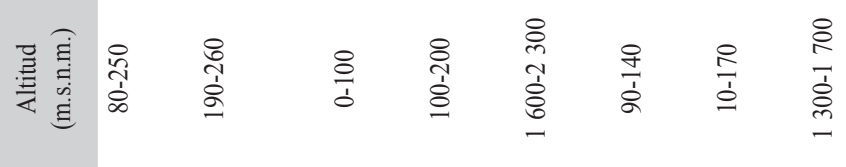

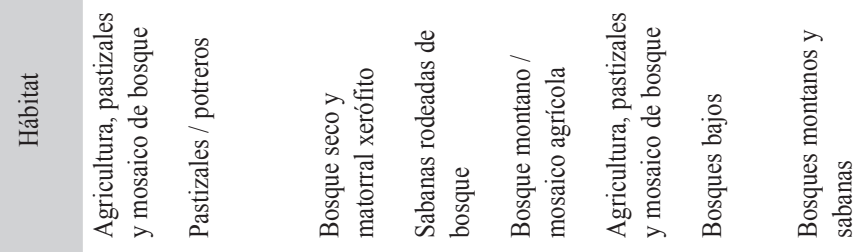

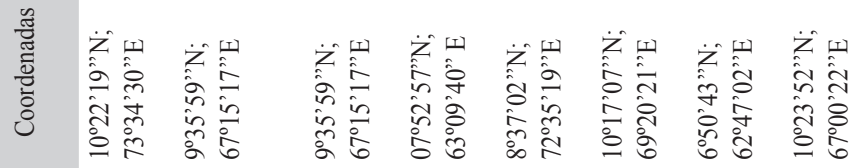

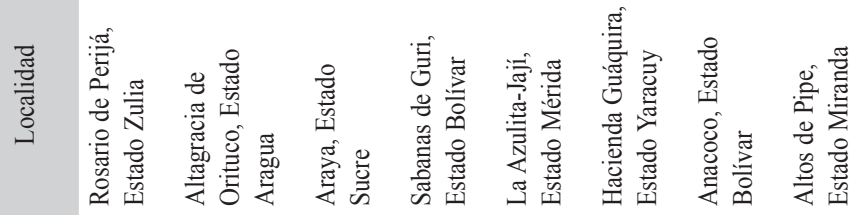

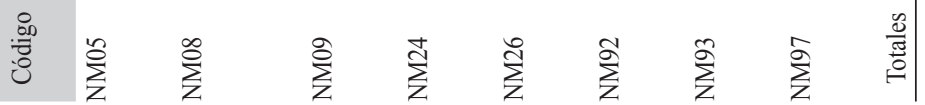



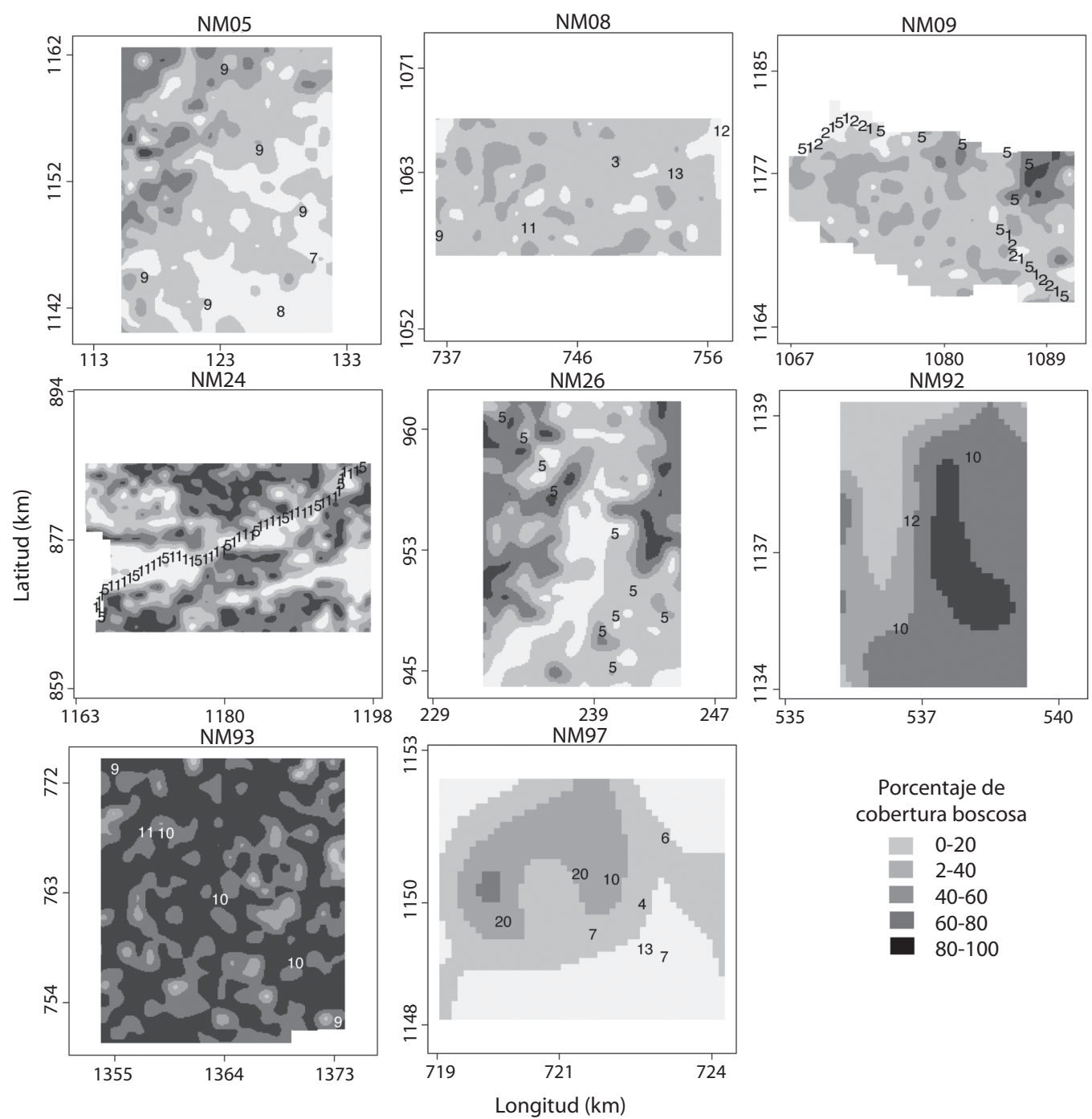

Porcentaje de cobertura boscosa

$\begin{array}{ll} & 0-20 \\ 2-40 \\ 40-60 \\ 60-80 \\ \square 0-100\end{array}$

Fig. 1. Disposición espacial de las trampas en cada uno de los ocho transectos estudiados. Los números dentro de los transectos indican el tamaño del grupo de trampas. Los ejes muestran las coordenadas en proyección UTM zona 19 norte. Fig. 1. Traps spatial arrangement in each transect studied. Numbers inside transects shows the size of each trap set. Axes show coordinates in UTM projection zone 19 north.

limpia y las bolsas fueron rotuladas con el código del transecto, el punto de muestreo, la fecha y la hora.

Los ejemplares de tres transectos (NM09, NM24 y NM26) recolectados en 2005 fueron identificados por Antonio A. De Ascençao (Universidad de los Andes, ULA, Venezuela) y se mantienen en su colección. El resto del material fue identificado por Ángel Solís
(Instituto Nacional de Biodiversidad-INBIO, en Costa Rica) utilizando las claves de Vazde-Mello et al. (2011) y las referencias bibliográficas contenidas en ellas. Este material se encuentra depositado en la colección del Centro de Ecología del Instituto Venezolano de Investigaciones Científicas (Registro Nacional de Colecciones Biológicas número 028). Los nombres de las especies fueron validados con 
la base de datos taxonómica The Scarabaeinae Research Network (ScarabNet 2011).

Riqueza de especies: Para cada uno de los cuatro tiempos de revisión $(24,48,72$ y 96hrs), calculamos el número de especies detectadas al incrementarse el número de trampas, siguiendo un orden aleatorio. Con el fin de discriminar si el crecimiento del número de especies estimadas fue debido al aumento en el número de trampas o por la extensión en el tiempo de muestreo, ajustamos un modelo STAR logarítmico (Species Time/Area Relationship) a las curvas de acumulación de especies (Ulrich 2006):

\section{Ec. $1 \mathrm{~S}=\beta_{0}+\beta_{1} \log (\mathrm{A})+\beta_{2} \log (\mathrm{T})+\beta_{3} \log (\mathrm{A}) \log (\mathrm{T})$}

donde $\beta_{0}$ es una constante, $A$ el área, entendida cómo el número de trampas, $T$ el esfuerzo de muestreo en horas, $\beta_{1}$ la pendiente de la relación especie-área, $\beta_{2}$ la pendiente de la relación especie-tiempo y $\beta_{3}$ el coeficiente de interacción entre ambas relaciones (Adler et al. 2005). Para cada transecto se ajustó el modelo completo con una regresión lineal y se eliminaron secuencialmente los términos redundantes hasta obtener un valor mínimo del criterio de información de Akaike (mejor conocido por sus siglas en inglés, AIC; Quinn $\&$ Keough 2002). Adicionalmente, utilizamos el método de Colwell et al. (2004) para estimar el promedio y los intervalos de confianza de las curvas de acumulación de especies por grupo de trampa dentro de los transectos NM08, NM92 NM93 y NM97 que contaban con 10 o más trampas por grupo.

Distribución de las abundancias: Para cada transecto graficamos la distribución de las abundancias de las especies en orden decreciente (RAD, Rank-Abundance Distribution, Mouillot \& Lepretre 2000). Los gráficos RAD permiten comparar muestras tomadas en localidades distintas que tengan pocas o ninguna especie en común (Fattorini 2005). La forma típica de estas curvas es un decrecimiento monotónico, aproximadamente log-normal, cuando el muestreo es completo, que puede ser ajustada con un modelo de preferencia de nicho (niche preemption). En este modelo, la abundancia esperada $a$ para una determina categoría de abundancia $a_{r}$ viene dada por:

$$
\text { Ec. } 2 a_{\mathrm{r}}=J(1-\alpha)^{(\mathrm{r}-1)}
$$

donde $J$ es el número total de individuos en la muestra y $\alpha$ el coeficiente que da la tasa de decaimiento de abundancia por categoría. En muestras diversas, con mayor equidad, $\alpha$ es menor que en muestras dominadas por una sola especie (Wilson 1991). Con el fin de evaluar si la distribución de abundancias ordenadas varía según el esfuerzo de muestreo, construimos el RAD basándonos en la cuenta acumulada de individuos de todas las trampas de un transecto al final de cada día de muestreo $(24,48,72$ y $96 \mathrm{hrs})$. Luego, para cada transecto graficamos el coeficiente $\alpha$ versus el esfuerzo de muestreo.

Efecto del tipo de cebo sobre la abundancia de especies: Para determinar si una especie tiene preferencias significativas por un tipo de cebo utilizamos el análisis de especies indicadoras propuesto por Dufrene \& Legendre (1997) que calcula el valor indicador de una especie como el producto de la fidelidad y abundancia relativa de una especie a un conjunto de muestras que forman un grupo. En nuestro caso, la fidelidad se define como la prevalencia de una especie entre las trampas de un grupo y la abundancia relativa la definimos como el número promedio de individuos por unidad de esfuerzo dentro de las trampas del grupo, dividido entre el número promedio de individuos por unidad de esfuerzo dentro de todas las trampas. Este análisis lo realizamos para los transectos NM09 y NM97 para los cuales agrupamos los diferentes tipos de cebos utilizados en dos grandes categorías: (i) restos animales en proceso de descomposición y (ii) heces. En nuestro caso, consideramos como un grupo a todas las trampas que utilizaron una categoría de cebo. 
Efecto del diseño muestreal sobre la composición de especies: Para evaluar el efecto del tipo de cebo, la disposición de los grupos de trampas y el esfuerzo de muestreo en la composición de especies capturadas en cada trampa, realizamos un análisis de varianza multivariado con permutaciones (PERMANOVA; Anderson 2001). Este análisis lo realizamos únicamente en dos transectos con grupos de trampas espacialmente bien definidos y variación en los tipos de cebos utilizados (NM09 y NM26; Cuadro 1). Para ello, construimos una matriz $S$, en donde cada celda era el número de individuos capturados por especie (columnas) en cada evento de revisión de una trampa (filas). Utilizamos el índice de Chao (Chao et al. 2005) como un estimador de la distancia biótica entre las filas de $S$ y con estos valores construimos una matriz de distancia, $D$. Luego, para hacer el PERMANOVA repartimos la varianza en $D$ entre las tres distintas fuentes de variación y ajustamos un modelo lineal. Debido a que los elementos de la matriz no son independientes, la significancia de cada factor fue calculada por medio de 1000 permutaciones de la matriz de datos original manteniendo la asignación de las muestras a las trampas (Tabachnick \& Fidell 2007).

Para evaluar de forma espacialmente explícita el efecto de la disposición de las trampas, aplicamos un correlograma de Mantel (Betts et al. 2009) a fin de calcular la autocorrelación espacial en la composición de especies en todos los transectos. A partir de una matriz St, en donde cada celda era el número de individuos capturados para cada especie (columnas) en cada trampa (filas), calculamos la matriz de distancias bióticas, $D$. El correlograma muestra el valor de la correlación de Mantel ( $r$ ) para los intervalos de distancia entre trampas, medidos con base en las coordenadas geográficas proyectadas para cada transecto.

\section{RESULTADOS}

Muestreo de escarabajos coprófagos: El esfuerzo de muestreo total fue de 47023 horas*trampa $(\mathrm{h} * \mathrm{t})$ y se capturaron 40337 ejemplares en los ocho transectos (Cuadro 1 y 2). Los dos transectos de menor longitud NM92 y NM97, representaron los extremos en cuanto esfuerzo de muestreo: NM92 tuvo el menor esfuerzo en $\mathrm{h}^{*} \mathrm{t}$ pero el segundo mayor número de individuos por unidad de esfuerzo, mientras que NM97 tuvo más de $9600 \mathrm{~h} *$ t pero un valor intermedio de individuos por unidad de esfuerzo. Ambos transectos tuvieron una riqueza similar, pero a juzgar por la altura y la cobertura boscosa, el muestreo no ha captado todas las especies que podrían estar presentes en NM92. Por otro lado, el transecto NM09 tuvo la menor cantidad de especies y el menor número de ejemplares por unidad de esfuerzo, mientras que NM93 tuvo una riqueza de especies mucho mayor que el resto de los transectos con el tercer mejor registro de ejemplares por unidad de esfuerzo. NM05 aportó casi un tercio del material recolectado.

Un $70 \%$ del total de los ejemplares fueron identificados a nivel de especie y el resto a nivel de morfoespecie, representando 23 géneros diferentes. El género más abundante fue Canthon con 18524 ejemplares y fue a su vez el que tuvo mayor número de especies (Cuadro 2, Anexo 1).

Riqueza de especies: Por lo general el coeficiente de regresión asociado al número de trampas $\left(\beta_{1}\right)$ fue mayor que el del número de horas de muestreo $\left(\beta_{2}\right)$, pero el término de interacción en algunos casos fue negativo (Cuadro $3)$. En estos casos, las curvas convergieron para valores altos de esfuerzo de muestreo (Fig. 2), lo cual siguiere que si se colocan pocas trampas es necesario dejarlas varios días, pero si se colocan muchas, el efecto de los días de muestreo no es tan importante. En los casos en que el término de interacción fue positivo (NM09, NM93) o no fue significativo (NM24 y NM08), el número de trampas y el tiempo de muestreo por trampas fueron complementarios y las curvas correspondientes a cada tiempo de revisión divergieron (Fig. 2, Cuadro 3). Sólo en uno de los transectos (NM08) el tiempo de muestreo no tuvo relevancia en la aparición de especies 
CUADRO 2

Tribus y géneros de Scarabaeinae recolectados

TABLE 2

Scarabaeinae tribus and genera collected

\begin{tabular}{|c|c|c|c|}
\hline Tribu & Género & $\mathrm{N}^{\mathrm{o}}$. ejemplares & $\mathrm{N}^{\mathrm{o}}$. especies/morfoespecies \\
\hline \multirow[t]{2}{*}{ Ateuchini } & Ateuchus & 3125 & 10 \\
\hline & Scatimus & 2 & 1 \\
\hline \multirow[t]{9}{*}{ Canthonini } & Agamopus & 13 & 1 \\
\hline & Anisocanthon & 316 & 3 \\
\hline & Canthon & 18524 & 20 \\
\hline & Canthonella & 300 & 1 \\
\hline & Deltochilum & 423 & 5 \\
\hline & Malagoniella & 9 & 1 \\
\hline & Pseudocanthon & 1334 & 4 \\
\hline & Scybalocanthon & 19 & 2 \\
\hline & Sylvicanthon & 227 & 1 \\
\hline \multirow[t]{3}{*}{ Coprini } & Canthidium & 6238 & 8 \\
\hline & Dichotomius & 2950 & 10 \\
\hline & Ontherus & 249 & 3 \\
\hline Coptodactylini & Uroxys & 932 & 12 \\
\hline Demarziellini & Bdelyropsis & 10 & 1 \\
\hline Oniticellini & Eurysternus & 921 & 10 \\
\hline \multirow[t]{2}{*}{ Onthophagini } & Digitonthophagus & 131 & 1 \\
\hline & Onthophagus & 4131 & 13 \\
\hline \multirow[t]{5}{*}{ Phanaeini } & Coprophanaeus & 300 & 4 \\
\hline & Diabroctis & 4 & 1 \\
\hline & Oxysternon & 27 & 1 \\
\hline & Phanaeus & 152 & 2 \\
\hline & Total & 40337 & 115 \\
\hline
\end{tabular}

CUADRO 3

Modelo de relación tiempo/área para evaluar la contribución del número de trampas y el tiempo de muestreo en el incremento en el número de especies detectadas

TABLE 3

Species Time/Area Relationship model to evaluate contribution of number of traps and sampling time in the increase of the number of species detected

\begin{tabular}{lcccccc} 
& $\beta_{0}$ & $\beta_{1}$ & $\beta_{2}$ & $\beta_{3}$ & AIC & $R^{2}$ adjustado \\
NM05 & 7.69 & 5.07 & 3.09 & -0.57 & 939.27 & 0.86 \\
NM08 & 6.79 & 4.52 & - & - & 724.40 & 1024.16 \\
NM09 & 4.34 & 1.72 & -0.13 & 0.35 & 1514.83 & 0.87 \\
NM24 & 2.63 & 4.21 & 2.08 & - & 687.54 & 0.84 \\
NM26 & 2.03 & 3.34 & 2.38 & -0.38 & 514.88 & 0.81 \\
NM92 & 3.68 & 4.66 & 4.18 & -0.54 & 968.01 & 0.84 \\
NM93 & 8.16 & 5.51 & 3.15 & 0.66 & 1099.04 & 0.92 \\
NM97 & 5.36 & 3.21 & 2.64 & -0.27 & 0.86 \\
\hline
\end{tabular}

$\beta_{0}$ : intercepto / intercept.

$\beta_{1}$ : coeficiente de regresión del logaritmo del número de trampas / regression coefficient of the logarithm of number of traps.

$\beta_{2}$ : coeficiente de regresión del logaritmo de la duración del muestro / regression coefficient of the logarithm of sampling duration.

$\beta_{3}$ : interacción entre número de trampas y duración del muestreo / interaction among number of traps and sampling duration. 


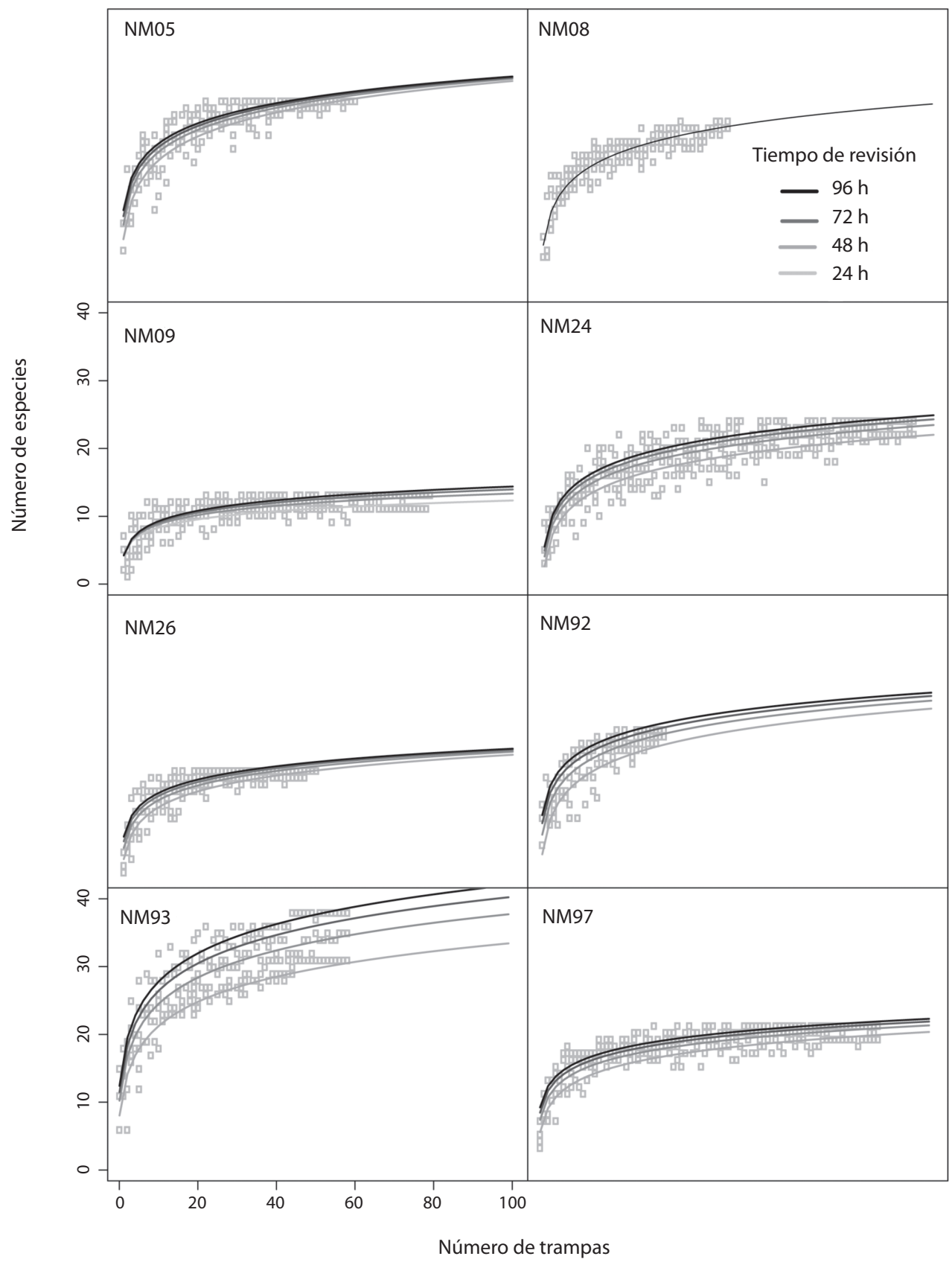

Fig. 2. Curvas de acumulación de especies. Las líneas muestran los valores ajustados para cada tiempo de revisión según el modelo STAR logarítmico.

Fig. 2. Species accumulation curves. Lines show adjusted value for each revision time following the STAR model. 
nuevas, sugiriendo (como se verá adelante) una alta dominancia en la composición de especies.

A pesar de que en algunas localidades el valor máximo de número de especies fue alcanzado luego de un esfuerzo de muestreo equivalente a 40-50 trampas, las curvas ajustadas tuvieron un aumento continuo, por lo que probablemente no se haya alcanzado el valor asintótico de riqueza de especies en ninguna de las localidades. Las curvas de acumulación de especies dentro de los grupos con más de 10 trampas mostraron por lo general una tendencia creciente, aunque en algunos casos (transectos NM97 y NM92) llegaron a valores estables con sólo cinco trampas (datos no se muestran).

Distribución de las abundancias: $\mathrm{La}$ distribución de las abundancias mantuvo una forma similar durante los cuatro días de muestreo para todos los transectos, pero entre el primer y el segundo día de muestreo se observó un aumento notable en los valores, probablemente debido a que el cebo fue más eficaz durante las primeras 48hrs (Fig. 3). En general,

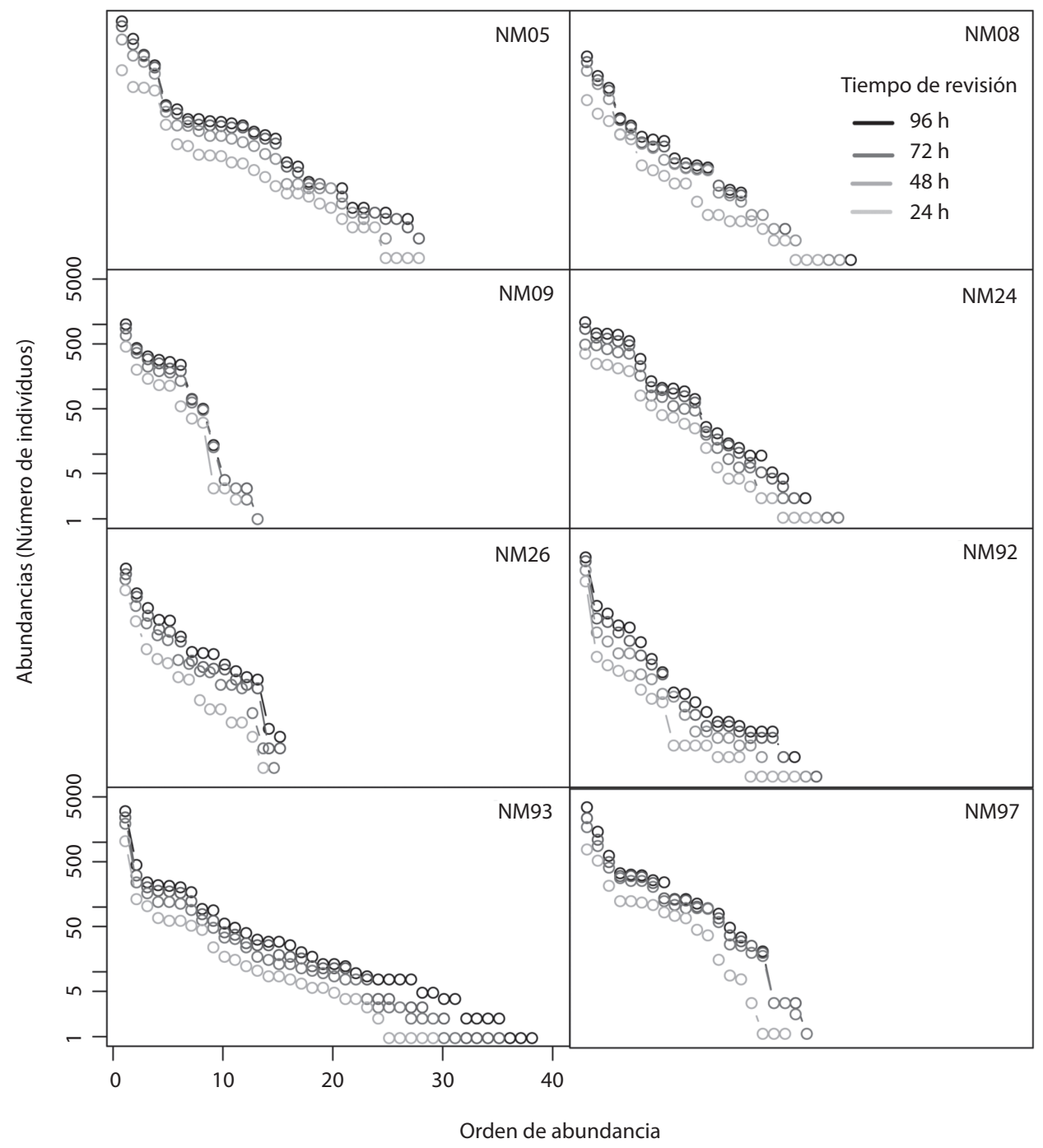

Fig. 3. Curvas de abundancias ordenadas para cada tiempo de revisión.

Fig. 3. Rank abundance curves by revision time. 
la composición de especies en las muestras fue haciéndose más equitativa (menor valor de $\alpha$ ) a medida que incrementó el esfuerzo de muestreo, estabilizándose los valores a partir de las 50hrs (Fig. 4). No obstante, al comparar el $\alpha$ de los transectos alrededor de las $100 \mathrm{hrs} \mathrm{de}$ muestreo, se observó que cinco poseen valores intermedios de equidad, (entre 0.3-0.35), pero en NM97 se requirió más del doble del esfuerzo de muestreo para obtener valores similares. Por el contrario, NM92 mantuvo valores muy altos de $\alpha$, indicando que las muestras estuvieron dominadas por una sola especie, mientras que NM24 fue el transecto con mayor equidad (Fig. 4).

Efecto del tipo de cebo sobre la abundancia de especies: La mayoría de las especies fueron capturadas con ambos tipos de cebos, aunque dos tercios mostraron abundancia relativa (ejemplares/esfuerzo de muestreo) mayor en cebos de heces. Por otro lado, la fidelidad a cada tipo de cebo estuvo muy relacionada con la frecuencia relativa o prevalencia de las especies en las muestras, la cual vario entre 1-82\%. Para las cinco especies que llegaron a un sólo tipo de cebo, la abundancia y frecuencia fue muy baja y por ello, no es posible discernir si se trata de preferencias reales o de un artefacto de muestreo. Según el análisis de valor indicador, sólo dos especies en NM09 y cinco en NM97 tuvieron una asociación significativa con una de las dos categorías de cebo: Coprophanaeus jasius y Deltochilum sp. [086] estuvieron asociados a cebos de tejido en descomposición, mientras que Onthophagus sp. [A23], Canthon politus, Canthidium sp. [089], Dichotomius protectus y Onthophagus curvicornis estuvieron asociados a cebos de heces (Cuadro 4).

Efecto del diseño muestreal sobre la composición de especies: La diferencia en la

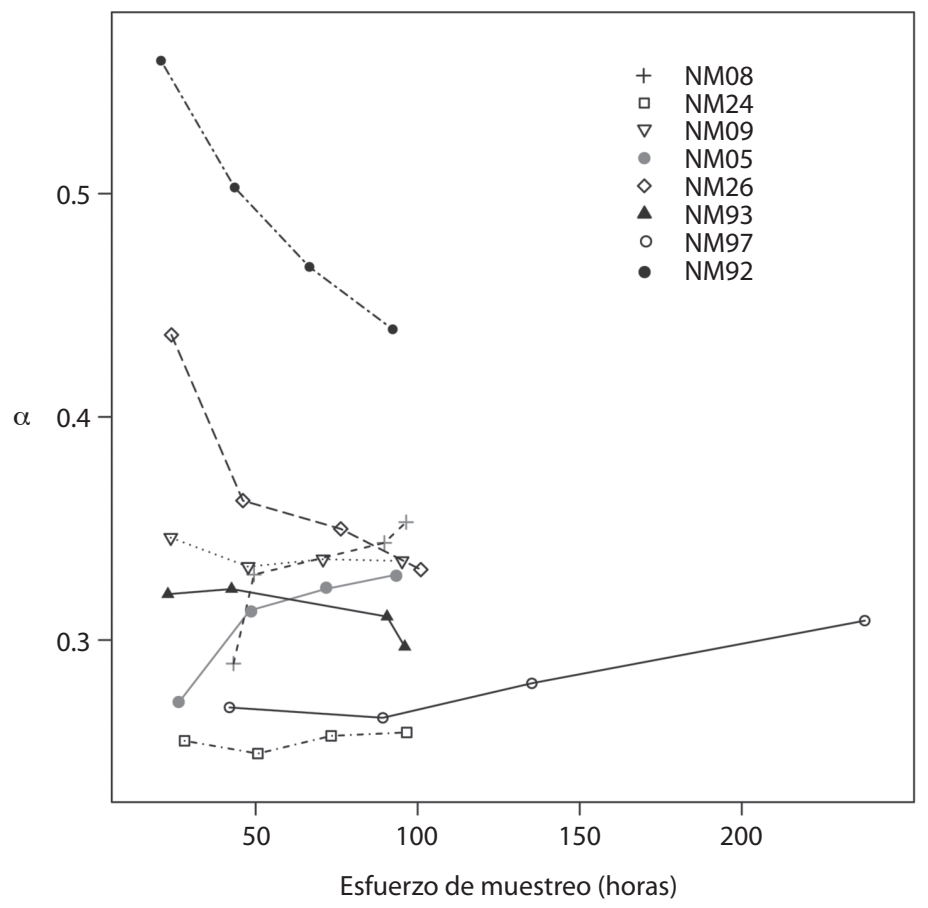

Fig. 4. Cambio en la composición de especies por efecto del esfuerzo de muestreo. El cambio en composición fue medido por medio de la tasa de decaimiento de las abundancias ordenadas $(\alpha)$.

Fig. 4. Change in species composition by sampling effort. The change in species composition was measure as the decline rate of rank abundance $(\alpha)$. 
CUADRO 4

Fidelidad (FR) y abundancia relativa (AR) de especies en distintos tipos de cebos

TABLE 4

Species' fidelity (FR) and abundance relative (AR) to different types of bait

\begin{tabular}{|c|c|c|c|c|c|c|c|}
\hline \multirow{2}{*}{ Especie } & \multicolumn{2}{|c|}{ (i) Materia descompuesta } & \multicolumn{2}{|c|}{ (ii) Heces } & \multirow{3}{*}{ Asociación } & \multirow{3}{*}{ VI } & \multirow{3}{*}{$\mathrm{P}$} \\
\hline & \multirow[t]{2}{*}{ FR } & \multirow[t]{2}{*}{$\mathrm{AR}$} & \multirow[t]{2}{*}{ FR } & \multirow[t]{2}{*}{$\mathrm{AR}$} & & & \\
\hline NM09 & & & & & & & \\
\hline Ateuchus sp. [A04] & 0.44 & 0.25 & 0.62 & 0.75 & Heces & 0.464 & n.s. \\
\hline Canthidium sp. [A07] & 0.75 & 0.49 & 0.54 & 0.51 & Desc & 0.369 & n.s. \\
\hline Canthon cyanellus & 0.69 & 0.61 & 0.43 & 0.39 & Desc & 0.420 & n.s. \\
\hline Canthon sp. [A14] & 0.56 & 0.47 & 0.22 & 0.53 & Desc & 0.265 & n.s. \\
\hline Canthon sp. [A15] & 0.50 & 0.61 & 0.30 & 0.39 & Desc & 0.307 & n.s. \\
\hline Coprophanaeus jasius & 0.19 & 1.00 & 0.00 & 0.00 & Desc & 0.188 & $<0.05$ \\
\hline Dichotomius agenor & 0.69 & 0.41 & 0.76 & 0.59 & Heces & 0.449 & n.s. \\
\hline Dichotomius coenosus & 0.00 & 0.00 & 0.08 & 1.00 & Heces & 0.081 & n.s. \\
\hline Ontophagus sp. [A22] & 0.12 & 0.09 & 0.30 & 0.91 & Heces & 0.271 & n.s. \\
\hline Ontophagus sp. [A23] & 0.06 & 0.03 & 0.38 & 0.97 & Heces & 0.366 & $<0.05$ \\
\hline Phanaeus prasinus & 0.00 & 0.00 & 0.03 & 1.00 & Heces & 0.027 & n.s. \\
\hline Pseudocanthon sp. [A25] & 0.06 & 0.53 & 0.03 & 0.47 & Desc & 0.033 & n.s. \\
\hline Uroxys sp. [A32] & 0.06 & 0.15 & 0.11 & 0.85 & heces & 0.092 & n.s. \\
\hline \multicolumn{8}{|l|}{ NM97 } \\
\hline Canthidium coerulescens & 0.16 & 0.26 & 0.33 & 0.74 & Heces & 0.243 & n.s. \\
\hline Canthidium lebasi & 0.37 & 0.42 & 0.57 & 0.58 & Heces & 0.328 & n.s. \\
\hline Canthidium sp. [089] & 0.53 & 0.38 & 0.76 & 0.62 & Heces & 0.472 & $<0.05$ \\
\hline Canthon cyanellus & 0.02 & 0.52 & 0.04 & 0.48 & Heces & 0.018 & n.s. \\
\hline Canthon juvencus & 0.02 & 1.00 & 0.00 & 0.00 & Desc & 0.023 & n.s. \\
\hline Canthon linearis & 0.09 & 0.56 & 0.05 & 0.44 & Desc & 0.052 & n.s. \\
\hline Canthon politus & 0.74 & 0.39 & 0.86 & 0.61 & Heces & 0.526 & $<0.05$ \\
\hline Canthon variabilis & 0.09 & 0.60 & 0.11 & 0.40 & Desc & 0.055 & n.s. \\
\hline Canthonella gomezi & 0.23 & 0.56 & 0.35 & 0.44 & Heces & 0.155 & n.s. \\
\hline Deltochilum sp. [086] & 0.30 & 0.70 & 0.15 & 0.30 & Desc & 0.211 & $<0.05$ \\
\hline Dichotomius agenor & 0.02 & 0.53 & 0.03 & 0.47 & Desc & 0.012 & n.s. \\
\hline Dichotomius protectus & 0.49 & 0.29 & 0.71 & 0.71 & Heces & 0.503 & $<0.01$ \\
\hline Eurysternus caribaeus & 0.16 & 0.39 & 0.23 & 0.61 & Heces & 0.139 & n.s. \\
\hline Eurysternus plebejus & 0.00 & 0.00 & 0.04 & 1.00 & Heces & 0.038 & n.s. \\
\hline Eurysternus superbus & 0.21 & 0.38 & 0.35 & 0.62 & Heces & 0.219 & n.s. \\
\hline Onthophagus curvicornis & 0.21 & 0.27 & 0.41 & 0.73 & Heces & 0.294 & $<0.05$ \\
\hline Onthophagus marginicollis & 0.02 & 0.55 & 0.03 & 0.45 & Desc & 0.013 & n.s. \\
\hline Onthophagus rhinophyllus & 0.30 & 0.42 & 0.47 & 0.58 & Heces & 0.274 & n.s. \\
\hline Uroxys [sp. 094] & 0.28 & 0.61 & 0.20 & 0.39 & Desc & 0.172 & n.s. \\
\hline Uroxys [sp. 091] & 0.16 & 0.44 & 0.25 & 0.56 & Heces & 0.142 & n.s. \\
\hline Uroxys [sp. 093] & 0.44 & 0.34 & 0.49 & 0.66 & Heces & 0.324 & n.s. \\
\hline
\end{tabular}

n.s. $=$ No significativo

VI: Índice de valor indicador para el cebo con el que la especie muestra mayor asociación / Indicator value index for the bait for which species showed major association.

p: Probabilidad de obtener un índice de valor indicador mayor al observado / probability to obtain an indicator value bigger than observed.

Nota / Note: Los números en corchetes corresponden a morfoespecies en la colección de referencia / Number inside brackets refers to morphospecies in the reference collection. 
composición de especies a lo largo del transecto fue explicada principalmente $(39-51 \%)$ por la disposición de las trampas dentro y entre grupos (grupos de trampas). Por el contrario, los tipos de cebo o las diferencias en el esfuerzo de muestreo sólo explicó 2-3\% (Cuadro 5).
Sin embargo, el análisis de PERMANOVA no resultó significativo para el transecto NM26, posiblemente debido a que las diferencias en la composición de especies entre trampas fue muy alta (valor promedio de disimilitud entre trampas 0.91).

\section{CUADRO 5}

Efecto del tipo de cebo, tamaño del grupo de trampas y esfuerzo de muestreo en las diferencias de composición de especies a lo largo de los transectos

TABLE 5

Effect of type of bait, size group of traps, and sampling effort in species composition differences through transects

\begin{tabular}{lcccccccc} 
& \multicolumn{3}{c}{ NM26 } & \multicolumn{3}{c}{ NM09 } \\
& GL & $\mathrm{F}$ & $\mathrm{R}^{2}$ & $\mathrm{p}$ & $\mathrm{GL}$ & $\mathrm{F}$ & $\mathrm{R}^{2}$ & $\mathrm{p}$ \\
Tipo de cebo & 1 & 4.16 & 0.02 & 0.2 & 1 & 8.53 & 0.03 & $<0.01$ \\
Grupo de trampas & 9 & 15.39 & 0.51 & 0.2 & 18 & 5.27 & 0.39 & $<0.01$ \\
Esfuerzo acumulado & 1 & 1.11 & 0.01 & 0.2 & 1 & 2.33 & 0.01 & $<0.01$ \\
Residuales & 130 & & 0.46 & & 138 & & 0.57 & \\
Total & 141 & & 1.00 & & 158 & & 1.00 & \\
\hline
\end{tabular}

GL: grados de libertad/degree of freedom.

$F$ : valor del estadístico $F$ de Fisher/Fisher's $F$ value.

$\mathrm{R}^{2}$ : proporción de varianza explicada/proportion of explained variance.

p: significancia/significance.

Para cuatro de los seis transectos de $40 \mathrm{~km}$, el correlograma de mantel presentó valores de autocorrelación positivos (valor de $r>0$ ) para distancias menores a $5 \mathrm{~km}$, y en el caso de NM09 y NM05 esta distancia se extiende hasta $10 \mathrm{~km}$ (Fig. 5). Los transectos mostraron autocorrelación negativa para distancias mayores a $10 \mathrm{~km}$. El valor de $r$ en distancias cortas fue mayor en NM26, que presenta un gradiente altitudinal marcado (Cuadro 1), y menor en NM24 y NM93, los cuales presentan una mayor cobertura arbórea (Fig. 1).

\section{DISCUSIÓN}

Evaluación del diseño muestreal: Existe un gran interés en desarrollar métodos de muestreo efectivos y prácticos para realizar inventarios a escalas geográficas amplias de un grupo tan diverso como lo son los escarabajos coprófagos (Lobo et al. 1997, Hortal et al. 2001, Lobo et al. 2007, Radtke et al. 2007,
Pierre et al. 2008). En este trabajo utilizamos la información sobre riqueza, abundancia y composición de especies para evaluar la combinación óptima de esfuerzo de muestreo, configuración espacial de las trampas y el tipo de cebo que permitiera generar estimaciones robustas y confiables de diversidad de especies. Un corolario que se deriva de los resultados es que cada uno de estos tres aspectos del diseño muestreal tuvo un efecto variable en los estimadores de riqueza, abundancia y composición. Por ejemplo, para obtener estimadores confiables de riqueza, el número de trampas fue el aspecto más importante, mientras que la duración del muestreo fue clave para obtener estimadores de abundancia precisos. Por otra parte, la disposición espacial de las trampas fue importante para evidenciar los patrones en la composición de especies.

Cuando se realizan inventarios a escalas geográficas amplias, además de balancear aspectos del diseño muestreal, se deben 


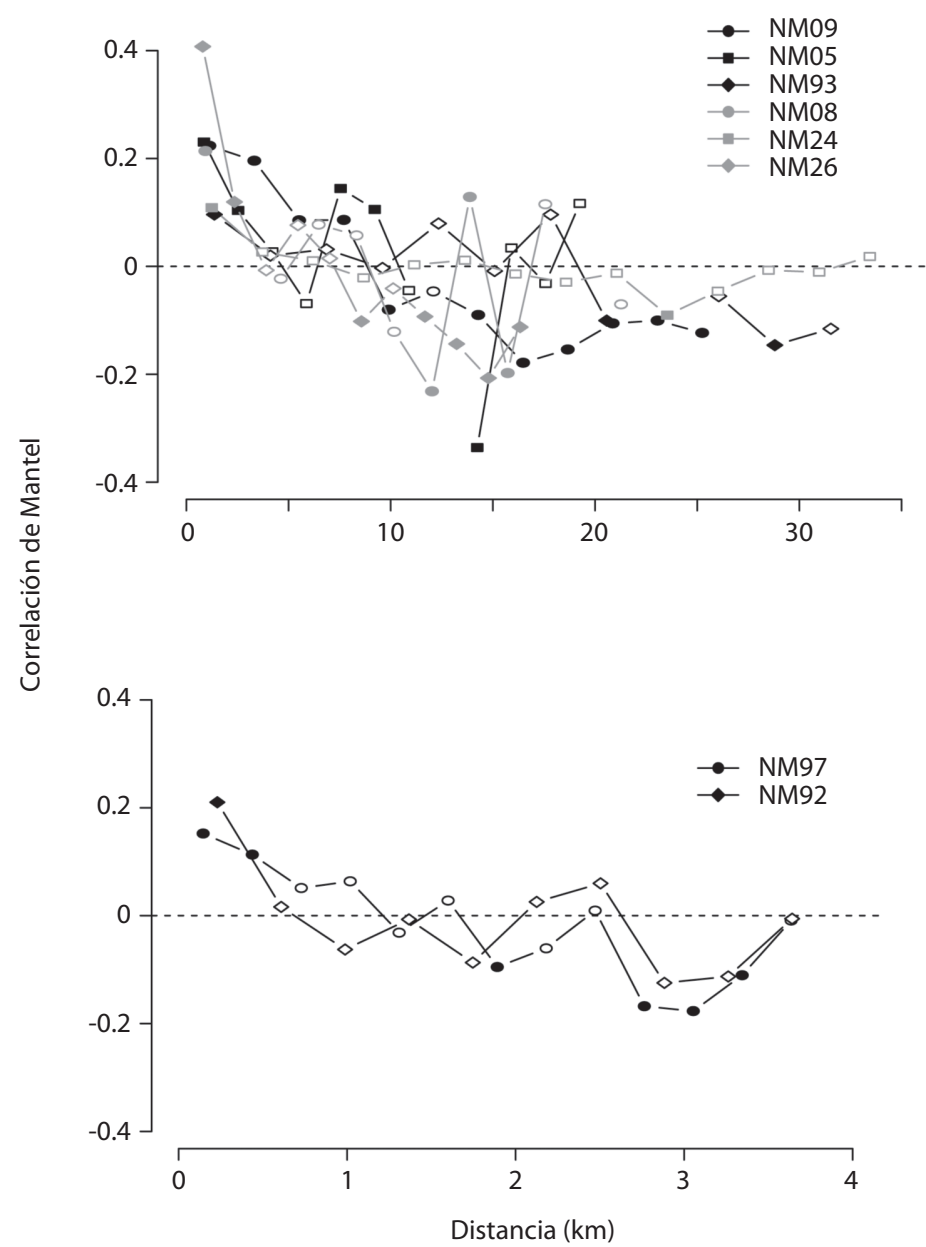

Fig. 5. Correlación espacial en la composición de especies. Figuras rellenas indican valores de $\mathrm{r}$ significativamente diferentes de cero. Figuras sin rellenar indican valores alrededor de cero.

Fig. 5. Spatial correlation on species composition. Close figures shown $r$ values significantly different of zero. Open figures shown values close to zero.

considerar los costos y las limitaciones logísticas. Por ejemplo, el costo material de colocar más trampas siempre es menor al costo de mantener al personal un día extra de trabajo. Sin embargo, el número de trampas que se pueden colocar y procesar en un día está limitado por el número de personas disponibles. Nuestra experiencia en el campo señala que 60-70 trampas es un número manejable tanto para la instalación, como para la revisión y procesamiento de las muestras si el equipo de trabajo está conformado por tres o cuatro personas.
Con base en los resultados obtenidos sugerimos que una estrategia balanceada consistiría en: (1) colocar entre 50-70 trampas en total para maximizar el número de especies detectadas, (2) muestrear al menos dos o tres días (48-72hrs) y colocar las trampas en grupos a lo largo del transecto para estimar de forma confiable la abundancia de las especies, (3) los grupos deben tener al menos 10 trampas para garantizar un buen registro de las especies de cada localidad, pero a la vez debe haber al menos siete grupos para estimar las diferencias 
en composición a lo largo del transecto y (4) los grupos de trampas deben estar separados por distancias mayores a $5-10 \mathrm{~km}$ para minimizar la autocorrelación espacial entre grupos.

En estudios regionales o restringidos a un determinado tipo de hábitat, la heterogeneidad espacial puede afectar las decisiones del diseño muestreal. Por ejemplo, el transecto NM93, dominado por vegetación boscosa presentó un número mayor de especies, las curvas de acumulación no se saturaron con el nivel de esfuerzo alcanzado y no se detecto un patrón espacial marcado. En este caso, además de la gran heterogeneidad espacial en la distribución de las especies, también se observó gran heterogeneidad en el comportamiento de las mismas, pues algunas no fueron capturadas sino hasta los últimos días de muestreo. Así, para obtener estimadores de riqueza confiables en este tipo de hábitat, se requeriría de un mayor esfuerzo de muestreo tanto en número de trampas como en la duración del muestreo. El caso de NM24 es muy similar, aunque el muestreo se hizo completamente al borde de la vegetación boscosa y se capturaron menos especies que en NM93. Por otra parte, los transectos NM09 y NM26 tuvieron un bajo número de especies, pero la composición de especies y la abundancia varió de forma marcada a lo largo de la misma, probablemente debido a la heterogeneidad espacial. En NM09 la heterogeneidad se debió a un gradiente de aridez que va de una zona de bosque seco hacia vegetación xerófita costera, mientras que en NM26 se trató de un gradiente de elevación en la Cordillera de Mérida. Así, en hábitats con alta heterogeneidad espacial, la distancia entre grupos de trampas debe ser mayor a $5 \mathrm{~km}$ para evitar los efectos negativos de la autocorrelación espacial en la estimación de patrones de composición de especies. Finalmente, los transectos dominados por paisajes ganaderos (NM05, NM08 y NM92), presentaron una rápida saturación en la curva de acumulación y las mayores abundancias totales, con una composición dominada por una o pocas especies. Este patrón puede ser explicado por la alta disponibilidad de recursos, y constituye el tipo de hábitat más fácil de muestrear, pues requieren pocas trampas por poco tiempo, en los cuales la riqueza, abundancia y composición de especies puede ser capturada bajo cualquier diseño muestreal.

Atracción de los diferentes tipos de cebo: Al contrario de lo reportado por Filgueiras et al. (2009), el uso de diferentes tipos de cebo tuvo un efecto menor en el número de especies detectadas y en la composición de especies. La efectividad de las trampas cebadas depende en gran medida de la atracción del cebo y puede disminuir con el paso de los días por la degradación, o aumentar debido a la fermentación (Flechtmann et al. 2009). Dado que en nuestros muestreos no hubo reemplazo de cebos, posiblemente la similitud observada en la composición de especies entre trampas cebadas con heces y las cebadas con tejido en descomposición, se deba a la pérdida de atracción de los mismos. Otra posible explicación es que el tamaño muestreal bajo o la abundancia baja de las especies más especializadas haya influido en el grado de detección de las especies (Kéry \& Plattner 2007). Así, lejos de indicar que el tipo de cebo es un factor poco relevante, nuestros resultados resaltan la importancia del mismo en el diseño muestreal. En este sentido, se requiere más estudios de campo, así como un análisis estadístico exhaustivo de la información existente, con el fin de determinar el ancho de la dieta de las especies o asociaciones concretas con un tipo de cebo (Flechtmann et al. 2009).

Si bien el tipo de cebo no pareció tener efecto en la composición, sí observamos diferencias en las abundancias para varias especies entre tipos de cebos (mayor abundancia en las trampas con heces) e incluso, logramos establecer las preferencias de un reducido número de especies. Los escarabajos coprófagos son esencialmente polífagos (Halffter \& Matthews 1966, Halffter \& Halffter 2009), aunque se ha documentado preferencias tróficas para algunas especies, usualmente asociadas con la disponibilidad de recursos. Por ejemplo, la mayoría de las especies son atraídas a heces de vertebrados con hábitos alimenticios similares, e.g. 
herbívoro, carnívoro, u omnívoro. Particularmente en las regiones tropicales de Suramérica se ha reportado una alta preferencia por estiércol de herbívoros, aunque las heces humanas también son atractivas para muchas especies de escarabajos coprófagos (Filgueiras et al. 2009). Sin embargo, a pesar de las observaciones en campo sobre preferencia de cebo y comportamiento, todavía el proceso de alimentación de los escarabajos coprófagos no se ha estudiado a fondo (Holter \& Scholtz 2007).

Nuestros hallazgos confirman evidencia previa sobre la efectividad de las heces humanas para atraer una gran variedad de especies de Scarabaeinae, lo cual tiene implicaciones importantes para la implementación de un muestreo a gran escala. Si bien muchas especies pueden tener una dieta amplia, el hecho de que sean atraídas en mayor grado por un cebo en particular influye en su detección. Por ejemplo varias especies de los géneros Coprophanaeus y Deltochilum tienen una marcada especialización por la carroña (Hanski 1983) y en nuestro caso, C. jasius y Deltochilum sp. [086] fueron encontrados casi exclusivamente en este tipo de cebo. Si las especies "escasas" son atraídas efectivamente a cebos alternativos, éstos deben ser imprescindibles en muestreos orientados al inventario de especies, pues son precisamente éstas las que más importancia tienen para estimar la riqueza de una región (Peck \& Howden 1984). En este sentido, varios autores han tomado en cuenta la gran diversidad trófica de los escarabajos coprófagos y han experimentado en sus muestreos con diferentes combinaciones de cebos e incluso, recomiendan el uso de trampas de interceptación, para capturar especies poco comunes (Peck \& Howden 1984, Filgueiras et al. 2009, Flechtmann et al. 2009).

Aplicabilidad de un diseño muestreal óptimo: Las recomendaciones realizadas en este trabajo fueron adoptadas para el primer muestreo nacional de escarabajos de Venezuela impulsado por NeoMapas en 2009. El muestreo sistemático con seis equipos de cuatro personas y 25 transectos de $40 \mathrm{~km}$ ubicados en diferentes regiones del país, permitió una cobertura geográfica amplia y una representación apropiada de la variabilidad ambiental de Venezuela en un período de dos meses de trabajo de campo (para más detalles ver www.neomapas. org). Este esfuerzo intensivo brindó la oportunidad idónea para re-evaluar la diversidad de este grupo taxonómico en Venezuela. Hasta este momento, la única lista de escarabajos coprófagos existente para Venezuela reseñaba 69 especies (Roze 1955, Favila 2005), aunque la base de datos de Scarabaeinae Research Network reporta 121 especies para el país (ScarabNet 2011). Ambas cifras representan un subestimado de la riqueza real de Scarabaeinae en Venezuela. De hecho, el presente estudio, con sólo ocho transectos muestreados, registró 115 especies/morfoespecies de escarabajos coprófagos y los análisis preliminares de NeoMapas predicen una riqueza entre 200 y 280 especies para todo el país (J. R. FerrerParis et al. 2012).

Existen pocos programas de muestreo de invertebrados tropicales de esta magnitud (Gardner et al. 2008, Janzen \& Hallwachs 2009) y la factibilidad y sostenibilidad de los mismos en el tiempo depende en gran medida de su efectividad y capacidad de detectar diferencias, cambios y tendencias. El presente trabajo propone una estrategia sencilla de optimización del diseño muestreal que puede ser aplicada a muestreos basados en trampas atrayentes y generalizada de manera sencilla a otros tipos de muestreo. Básicamente, recomendamos identificar los elementos del diseño muestreal que afectan directamente el esfuerzo de muestreo (número de trampas, número de recolectores, duración del muestreo, etc.) y la distribución espacial del mismo (ubicación de las trampas o unidades elementales de muestreo a lo largo del transecto), para luego evaluar su efecto sobre las variables de interés, según los métodos de estimación seleccionados (curvas de acumulación, estimadores de abundancia o disimilitud, etc.). Existen otras estrategias alternativas y complementarias para optimizar los programas de inventario, por ejemplo, incorporando métodos de muestreo o modelos analíticos para estimar la probabilidad 
de detección de los individuos junto con las variables de interés (Kéry \& Plattner 2007, Rodríguez et al. 2012), o realizando pruebas y simulaciones de poder estadístico (Meyer et al. 2010). Cualquiera de estas estrategias permitirá hacer proyecciones realistas del alcance de los programas de inventario y monitoreo que se pretendan establecer en regiones tropicales.

\section{AGRADECIMIENTOS}

Este trabajo fue financiado por Conservación Internacional Venezuela, el Instituto Venezolano de Investigaciones Científicas (IVIC) y el Convenio de Cooperación entre Total Venezuela, S.A. e IVIC, en el marco de la Ley Orgánica de Ciencia, Tecnología e Innovación. José Piñango, Tatiana Caldera, Sergio Zambrano, Carmen Liendo, Diana Duque, Antoine Escalas, Linda Rosen, Janet Van Zoeren, Rebecca Sears, Ruben Badia, Leonardo Badia, Louise Rodgers, Javier Gutierrez-Illán, Luis Martínez, Tatjana Good, Mariana Alarcón, María Eugenia Losada, Fernando Rey, Iván Lau, Jesús Gutiérrez, Luis Argenis Martínez, Ileana Herrera, Oliany Cisneros, Alejandra Dos Ramos, Wence Hernández, Douglas Mora. Pío Colmenares, Julia Álvarez, Cecilia Lozano, Nora Paris, Gustavo Bracho, Luis Mujica, Helios Martínez y Yelitza Velásquez participaron en los muestreos. Tres árbitros anónimos contribuyeron con sugerencias para mejorar el manuscrito original.

\section{RESUMEN}

El presente trabajo propone una estrategia sencilla de optimización del diseño muestreal para escarabajos coprófagos que puede ser aplicada a muestreos basados en trampas atrayentes en amplias escalas geográficas y generalizada a otros tipos de muestreo. Para ello, analizamos muestras colectadas en ocho localidades con hábitats contrastantes y diferentes características del muestreo entre 2006-2008. Se capturaron 40337 ejemplares (115 especies/ morfoespecies, de 23 géneros). El número de trampas fue el aspecto del muestreo más importante para obtener estimadores de riqueza confiable. El tiempo de muestreo para generar estimadores precisos de abundancia y la disposición espacial de las trampas para captar adecuadamente la diferencias en composición entre localidades. Sugerimos que una estrategia óptima de muestreo para obtener estimadores precisos de riqueza, abundancia y diversidad consistiría en: (1) colocar 50-70 trampas para maximizar el número de especies detectadas, (2) muestrear entre 48 y 72 hrs y colocar grupos de trampas a lo largo de un transecto para estimar confiablemente la abundancia de las especies, (3) colocar siete grupos de al menos 10 trampas para registrar adecuadamente la composición de cada localidad y (4) separar los grupos de trampas por distancias mayores a 5-10km para minimizar la autocorrelación espacial.

Palabras clave: composición de especies, inventario de biodiversidad, muestreo sistemático, NeoMapas, riqueza.

\section{REFERENCIAS}

Adler, P.B., E.P. White, W.K. Lauenroth, D.M. Kaufman, A. Rassweiler \& J.A. Rusak. 2005. Evidence for a general species-time-area relationship. Ecology 86: 2032-2039.

Ahumada, J.A., C.E.F. Silva, K. Gajapersad, C. Hallam, J. Hurtado, E. Martin, A. McWilliam, B. Mugerwa, T. O'Brien, F. Rovero, D. Sheil, W.R. Spironello, N. Winarni \& S.J. Andelman. 2011. Community structure and diversity of tropical forest mammals: Data from a global camera trap network. Philos. Trans. R. Soc. Lond. Ser. B. 366: 2703-2711.

Anderson, M.J. 2001. A new method for non-parametric multivariate analysis of variance. Austral Ecol. 26: $32-46$

BBS. 2010. North American Breeding Bird Survey. The USGS Patuxent Wildlife Research Center. The Canadian Wildlife Service, National Wildlife Research Center MD. (Consultado: 21/07/2010, http://www. pwrc.usgs.gov/BBS/).

Betts, M.G., L.M. Ganio, M.M.P. Huso, N.A. Som, F. Huettmann, J. Bowman \& B.A. Wintle. 2009. Comment on "Methods to account for spatial autocorrelation in the analysis of species distributional data: A review". Ecography 32: 374-378.

Buzas, M.A. \& L.A.C. Hayek. 1996. Biodiversity resolution: An integrated approach. Biodiversity Lett. 3: 40-43.

Cardoso, P., P.A.V. Borges \& J.A. Veech. 2009. Testing the performance of beta diversity measures based on incidence data: The robustness to undersampling. Divers. Distrib. 15: 1081-1090.

Cardoso, P., S.S. Henriques, C. Gaspar, L.C. Crespo, R. Carvalho, J.B. Schmidt, P. Sousa \& T. Szüts. 2008. Rapid biodiversity assessment of spiders (Araneae) using semi-quantitative sampling: A case study in a Mediterranean forest. Insect Conserv. Diver. 1: 71-84.

Carr III, A. \& A.C. de Stoll. 2005. Biological monitoring in the Selva Maya. US Man and the Biosphere Program, 
Tropical Ecosystem Directorate \& Wildlife Conservation Society, Gainesville, Florida, EEUU.

Chao, A., R.L. Chazdon, R.K. Colwell \& T.J. Shen. 2005. A new statistical approach for assessing similarity of species composition with incidence and abundance data. Ecol. Lett. 8: 148-159.

Colwell, R.K. 2009. Biodiversity: Concepts, patterns, and measurement, p. 257-263. In S.A. Levin (ed.). The Princeton Guide to Ecology. Princeton University, Princeton, Nueva Jersey, EEUU.

Colwell, R.K. \& J.A. Coddington. 1994. Estimating terrestrial biodiversity through extrapolation. Philos. Trans. R. Soc. Lond. Ser. B. 345: 101-118.

Colwell, R.K., C.X. Mao \& J. Chang. 2004. Interpolating, extrapolating, and comparing incidence-based species accumulation curves. Ecology 85: 2717-2727.

Dufrene, M. \& P. Legendre. 1997. Species assemblages and indicator species: The need for a flexible asymetrical approach. Ecol. Monogr. 67: 345-366.

Fattorini, S. 2005. A simple method to fit geometric series and broken stick models in community ecology and island biogeography. Acta Oecol. 28: 199-205.

Favila, M.E. 2005. Alpha and beta diversity of dung beetles (Scarabaeinae) at Los Tuxtlas, Mexico, p. 209-219. In G. Halffter, J. Soberón, P. Koleff \& A. Melic (eds.). Sobre diversidad biológica: El significado de las diversidades alfa, beta y gamma. Monografias Tercer Milenio, Zaragoza, España.

Ferrer-Paris, J.R., J.P. Rodríguez, T. Good, A. SánchezMercado, K.M. Rodríguez-Clark, G.A. Rodríguez \& A. Solís. 2012. Systematic, large-scale national biodiversity surveys: NeoMaps as a model for tropical region. Divers. Distrib. DOI: 10.1111/ddi.12012.

Filgueiras, B.K.C., C.N. Liberal II, C.D.M. Aguiar, M.I.M. Hernández \& L. Lannuzzi. 2009. Attractivity of omnivore, carnivore and herbivore mammalian dung to Scarabaeinae (Coleoptera, Scarabaeidae) in a tropical Atlantic rainforest remnant. Rev. Bras. Entomol. 53: 422-427.

Fisher, B.L. 1999. Improving inventory efficiency: A case study of leaf-litter ant diversity in Madagascar. Ecol. Appl. 9: 714-731.

Flechtmann, C.A.H., V. Gomes-Tabes \& I. Quintero. 2009. Influence of carrion smell and rebaiting time on the efficiency of pitfall traps to dung beetle sampling. Entomol. Exp. Appl. 132: 211-217.

Gardner, T.A., J. Barlow, I.S. Araujo, T.C. Avila-Pires, A.B. Bonaldo, J.E. Costa, M.C. Esposito, L.V. Ferreira, J. Hawes, M.I.M. Hernandez, M.S. Hoogmoed, R.N. Leite, N.F. Lo-Man-Hung, J.R. Malcolm, M.B. Martins, L.A.M. Mestre, R. Miranda-Santos, W.L. Overal, L. Parry, S.L. Peters, M.A. Ribeiro-Junior, M.N.F. da Silva, C. da Silva Motta \& C.A. Peres. 2008. The cost-effectiveness of biodiversity surveys in tropical forests. Ecol. Lett. 11: 139-150.

Gotelli, N.J. \& R.K. Colwell. 2001. Quantifying biodiversity: Procedures and pitfalls in the measurement and comparison of species richness. Ecol. Lett. 4: 379-391.

Halffter, G. \& M.E. Favila. 1997. The Scarabaeinae (Insecta: Coleoptera) an animal group for analysing, inventoring and monitoring biodiversity in tropical rainforest and modified landscapes. Biol. Int. 27: $15-21$

Halffter, G. \& V. Halffter. 2009. Why and where coprophagous beetles (Coleoptera: Scarabaeinae) eat seeds, fruits or vegetable detritus. Boletín de la S.E.A. 45: $1-22$.

Halffter, G. \& E.G. Matthews. 1966. The natural history of dung beetles of the subfamily Scarabaeinae (Coleoptera, Scarabaeidae). Folia Ent. Mex. 12-14: 1-312.

Hanski, I. 1983. Distributional ecology and abundance of dung and carrion-feeding beetles (Scarabaeidae) in tropical rain forests in Sarawak, Borneo. Acta Zool. Fenn. 167: 1-45.

Holter, P. \& C.H. Scholtz. 2007. What do dung beetles eat? Ecol. Entomol. 32: 690-697.

Hortal, J., J.M. Lobo \& F. Martín-Piera. 2001. Forecasting insect species richness scores in poorly surveyed territories: The case of the Portuguese dung beetles (Col. Scarabaeinae). Biod. Conserv. 10: 1343-1367.

Janzen, D.H. \& W. Hallwachs. 2009. Dynamic database for an inventory of the macrocaterpillar fauna, and its food plants and parasitoids, of Area de Conservacion Guanacaste (ACG), northwestern Costa Rica (nn-SRNP-nnnnn voucher codes). (Consultado: 01/11/2011, http://janzen.sas.upenn.edu).

Jiménez-Valverde, A. \& J.M. Lobo. 2005. Determining a combined sampling procedure for a reliable estimation of Araneidae and Thomosidae assemblages (Arachnida, Araneae). J. Arachnol. 33: 33-42.

Kéry, M. \& M. Plattner. 2007. Species richness estimation and determinants of species detectability in butterfly monitoring programmes. Ecol. Entomol. 32: 53-61.

Kéry, M. \& H. Schmid. 2004. Monitoring programs need to take into account imperfect species detectability. Basic Appl. Ecol. 5: 65-73.

Larsen, T.H. \& A. Forsyth. 2005. Trap spacing and transect design for dung beetle biodiversity studies. Biotropica $37: 322-325$.

Lobo, J.M., E. Chehlarov \& B. Guéorguiev. 2007. Variation in dung beetle (Coleoptera: Scarabaeoidea) assemblages with altitude in the Bulgarian Rhodopes Mountains: A comparison. Eur. J. Entomol. 104: 489-495.

Lobo, J.M., I. Sanmartin \& F. Martín-Piera. 1997. Diversity and spatial turnover of dung beetles (Coleoptera: 
Scarabaeoidae) communities in a protected area of South Europe (Doñana National Park, Huelva, Spain). Elytron 11: 71-88.

Margules, C.R. \& R.L. Pressey. 2000. Systematic conservation planning. Nature. 405: 243-253.

Meyer, C.F.J., L.M.S. Aguiar, L.F. Aguirre, J. Baumgarten, F.M. Clarke, J.F. Cosson, S.E. Villegas, J. Fahr, D. Faria, N. Furey, M. Henry, R. Hodgkison, R.K.B. Jenkins, K.G. Jung, T. Kingston, T.H. Kunz, M.C. MacSwiney Gonzalez, I. Moya, J.M. Pons, P.A. Racey, K. Rex, E.M. Sampaio, K.E. Stoner, C.C. Voigt, D.V. Staden, C.D. Weise \& E.K.V. Kalko. 2010. Longterm monitoring of tropical bats for anthropogenic impact assessment: Gauging the statistical power to detect population change. Biol. Conserv. 143: 2797-2807.

Nichols, E., S. Spector, J. Louzada, T. Larsen, S. Amequita \& M.E. Favila. 2008. Ecological functions and ecosystem services provided by Scarabaeinae dung beetles. Biol. Conserv. 6: 1461-1474.

Noriega, J.A. \& G. Fagua. 2009. Monitoreo de escarabajos coprófagos (Coleoptera: Scarabaeidae) en la región neotropical, p. 165-188. In A. Acosta, G. Fagua \& A.M. Zapata (eds.). Técnicas de campo en ambientes tropicales, manual para el monitoreo en ecosistemas acuáticos y artrópodos terrestres. Pontificia Universidad Javeriana, Bogotá, Colombia.

Peck, S.B. \& H.F. Howden. 1984. Response of a dung beetle guild to different sizes of dung bait in a Panamanian rainforest. Biotropica 16: 235-238.

Pierre, J.R., J.P. Lumaret \& J.D. Lebreton. 2008. Spatial and temporal variation of mountain dung beetle assemblages and their relationships with environmental factors (Aphodiinae: Geotrupinae: Scarabaeinae). Ann. Entomol. Soc. Am. 101: 58-62.

Quinn, G.P. \& M.J. Keough. 2002. Experimental design and data analysis for biologists. Cambridge University, Cambridge, Reino Unido.

Radtke, M.G., C.R.V. da Fonseca \& G.B. Williamson. 2007. The old and young Amazon: Dung beetle biomass, abundance, and species diversity. Biotropica 39: 725-730.

Radtke, M.G., C.R.V. da Fonseca \& G.B. Williamson. 2010. Dung beetle communities: A neotropical-north temperate comparison. Neotrop. Entomol. 39: 19-27.

Rodríguez, G.A., J.P. Rodríguez, J.R. Ferrer-Paris \& A. Sánchez-Mercado. 2012. A nation-wide standardized bird survey scheme for Venezuela. Wilson J. Ornithol. 124: 230-244.

Rodríguez, J.P. \& C.J. Sharpe. 2002. NeoMaps: The Neotropical biodiversity mapping initiative. Cotinga 17 : $13-14$.
Rohr, J.R., C.G. Mahan \& K.C. Kim. 2007. Developing a monitoring program for invertebrates: Guidelines and a case study. Cons. Biol. 21: 422-433.

Roze, J.A. 1955. Lista preliminar de la familia Scarabaeinae sensu lato (Coleoptera) de Venezuela. B. Mus. Cien. Nat. I: 39-63.

ScarabNet. 2011. Scarabaeinae Research Network. National Science Foundation's Research Coordination Network program. National Aeronatics and Space Administration. (Consultado: 02 mayo 2011, http://216.73.243.70/scarabnet/).

Schieck, J. \& H.A. Stelfox. 2006. Alberta biodiversity monitoring program-monitoring effectiveness of sustainable forest management planning. Environ. Monit. Assess. 121: 33-46.

Spector, S. 2006. Scarabaeine dung beetles (Coleoptera: Scarabaeidae: Scarabaeinae): An invertebrate focal taxon for biodiversity research and conservation. Coleopt. Bull. 5: 71-83.

Tabachnick, B.G. \& L.S. Fidell. 2007. Using multivariate statistics. Allyn \& Bacon, Boston, Massachusetts, EEUU.

Ulrich, W. 2006. Decomposing the process of species accumulation into area dependent and time dependent parts. Ecol. Res. 21: 578-585.

Vaz-de-Mello, F.Z., W.D. Edmonds, F.C. Ocampo \& P. Schoolmeesters. 2011. A multilingual key to the genera and subgenera of the subfamily Scarabaeinae of the New World (Coleoptera: Scarabaeidae). Zootaxa 2854: 1-73.

Vennila, S. \& D. Rajagopal. 1999. Optimum sampling effort for study of tropical ground beetles (Carabidae: Coleoptera) using pitfall traps. Curr. Sci. 77: 281-283.

Villarreal, H., M. Álvarez, S. Córdoba, F. Escobar, G. Fagua, F. Gast, H. Mendoza, M. Ospina \& A.M. Umaña. 2004. Manual de métodos para el desarrollo de inventarios de biodiversidad. Programa de Inventarios de Biodiversidad. Instituto de Investigación de Recursos Biológicos Alexander von Humboldt, Bogotá, Colombia.

Walpole, M.J. \& I.R. Sheldon. 1999. Sampling butterflies in tropical rainforest: An evaluation of a transect walk method. Biol. Conserv. 87: 85-91.

Ward, D.F. \& M.C. Lariviere. 2004. Terrestrial invertebrate surveys and rapid biodiversity assessment in New Zealand: Lessons from Australia. N. Z. J. Ecol. 28: 151-159.

Watson, D.M. 2010. Optimizing inventories of diverse sites: Insights from Barro Colorado island birds. Methods Ecol. Evol. 1: 280-291.

Wilson, J.B. 1991. Methods for fitting dominance/diversity curves. J. Veg. Sci. 2: 35-46. 
ANEXO

Número de ejemplares capturados por especie de escarabajos coprófagos. Los números en corchetes corresponden a los códigos provisionales de las morfoespecies en la colección de referencia

APPENDIX

Number of individuals sampled per dung beetles species. Number inside square brackets refers to morphospecies codes in the reference collection

\begin{tabular}{|c|c|c|c|c|c|c|c|c|}
\hline Especie/morfoespecie & NM05 & NM08 & NM09 & NM24 & NM26 & NM92 & NM93 & NM97 \\
\hline Agamopus sp. [066] & 2 & 11 & 0 & 0 & 0 & 0 & 0 & 0 \\
\hline Anisocanthon sericinus Harold, 1868 & 0 & 0 & 0 & 0 & 0 & 0 & 31 & 0 \\
\hline Anisocanthon sp. [A01] & 0 & 0 & 0 & 68 & 0 & 0 & 0 & 0 \\
\hline Anisocanthon villosus (Harold, 1868 & 0 & 0 & 0 & 0 & 0 & 217 & 0 & 0 \\
\hline Ateuchus sp. [020] & 0 & 0 & 0 & 0 & 0 & 0 & 99 & 0 \\
\hline Ateuchus sp. [028] & 0 & 0 & 0 & 0 & 0 & 0 & 8 & 0 \\
\hline Ateuchus sp. [039] & 0 & 0 & 0 & 0 & 0 & 0 & 4 & 0 \\
\hline Ateuchus sp. [047] & 0 & 2 & 0 & 0 & 0 & 0 & 0 & 0 \\
\hline Ateuchus sp. [048] & 0 & 3 & 0 & 0 & 0 & 0 & 0 & 0 \\
\hline Ateuchus sp. [072] & 6 & 0 & 0 & 0 & 0 & 2413 & 0 & 0 \\
\hline Ateuchus sp. [074] & 5 & 0 & 0 & 0 & 0 & 0 & 0 & 0 \\
\hline Ateuchus sp. [A02] & 0 & 0 & 0 & 0 & 57 & 0 & 0 & 0 \\
\hline Ateuchus sp. [A03] & 0 & 0 & 0 & 88 & 0 & 0 & 0 & 0 \\
\hline Ateuchus sp. [A04] & 0 & 0 & 440 & 0 & 0 & 0 & 0 & 0 \\
\hline Bdelyropsis venezuelensis Howden, 1976 & 0 & 0 & 0 & 0 & 0 & 10 & 0 & 0 \\
\hline Canthidium coerulescens Balthasar,1939 & 0 & 0 & 0 & 0 & 0 & 0 & 0 & 120 \\
\hline Canthidium lebasi Harold, 1867 & 2452 & 154 & 0 & 0 & 0 & 435 & 50 & 285 \\
\hline Canthidium sp. [019] & 0 & 0 & 0 & 0 & 0 & 0 & 5 & 0 \\
\hline Canthidium sp. [041] & 0 & 0 & 0 & 0 & 0 & 0 & 2 & 0 \\
\hline Canthidium sp. [089] & 0 & 0 & 0 & 0 & 0 & 0 & 0 & 1326 \\
\hline Canthidium sp. [A05] & 0 & 0 & 0 & 0 & 292 & 0 & 0 & 0 \\
\hline Canthidium sp. [A06] & 0 & 0 & 0 & 101 & 0 & 0 & 0 & 0 \\
\hline Canthidium sp. [A07] & 0 & 0 & 1016 & 0 & 0 & 0 & 0 & 0 \\
\hline Canthon cyanellus LeConte, 1859 & 121 & 0 & 267 & 0 & 0 & 20 & 2 & 23 \\
\hline Canthon juvencus Harold, 1868 & 70 & 118 & 0 & 0 & 0 & 41 & 21 & 1 \\
\hline Canthon linearis Schmidt, 1920 & 0 & 452 & 0 & 0 & 0 & 0 & 42 & 87 \\
\hline Canthon lituratus Germar 1813 & 200 & 79 & 0 & 0 & 0 & 0 & 0 & 0 \\
\hline Canthon mutabilis Lucas, 1857 & 4489 & 72 & 0 & 0 & 0 & 0 & 0 & 0 \\
\hline Canthon politus Harold, 1868 & 0 & 0 & 0 & 0 & 1188 & 0 & 0 & 3150 \\
\hline Canthon quadriguttatus (Olivier,1789) & 0 & 0 & 0 & 0 & 0 & 0 & 5 & 0 \\
\hline Canthon septemmaculatus Latreille, 1811 & 90 & 1389 & 0 & 0 & 0 & 0 & 0 & 0 \\
\hline Canthon simulans Martínez, 1950 & 0 & 0 & 0 & 0 & 0 & 0 & 8 & 0 \\
\hline Canthon sp. [025] & 0 & 0 & 0 & 0 & 0 & 0 & 1 & 0 \\
\hline Canthon sp. [A08] & 0 & 0 & 0 & 696 & 0 & 0 & 0 & 0 \\
\hline Canthon sp. [A09] & 0 & 0 & 0 & 662 & 0 & 0 & 0 & 0 \\
\hline Canthon sp. [A10] & 0 & 0 & 0 & 282 & 0 & 0 & 0 & 0 \\
\hline Canthon sp. [A11] & 0 & 0 & 0 & 686 & 0 & 0 & 0 & 0 \\
\hline Canthon sp. [A12] & 0 & 0 & 0 & 20 & 0 & 0 & 0 & 0 \\
\hline Canthon sp. [A13] & 0 & 0 & 0 & 1 & 0 & 0 & 0 & 0 \\
\hline Canthon sp. [A14] & 0 & 0 & 239 & 0 & 0 & 0 & 0 & 0 \\
\hline Canthon sp. [A15] & 0 & 0 & 288 & 0 & 0 & 0 & 0 & 0 \\
\hline Canthon triangularis Drury, 1770 & 0 & 0 & 0 & 0 & 0 & 0 & 3194 & 0 \\
\hline
\end{tabular}


ANEXO / APPENDIX (Continucación / Continued)

\begin{tabular}{|c|c|c|c|c|c|c|c|c|}
\hline Especie/morfoespecie & NM05 & NM08 & NM09 & NM24 & NM26 & NM92 & NM93 & NM97 \\
\hline Canthon variabilis Martínez,1948 & 26 & 0 & 0 & 0 & 0 & 1 & 474 & 19 \\
\hline Canthonella gomezi Halffter \& Martínez, 1968 & 0 & 0 & 0 & 0 & 0 & 0 & 0 & 300 \\
\hline Coprophanaeus dardanus Macleay, 1819 & 0 & 5 & 0 & 0 & 0 & 0 & 2 & 0 \\
\hline Coprophanaeus gamezi Arnaud, 2002 & 231 & 12 & 0 & 0 & 0 & 19 & 0 & 0 \\
\hline Coprophanaeus jasius (Olivier,1789) & 0 & 0 & 4 & 9 & 0 & 0 & 13 & 0 \\
\hline Coprophanaeus telamon (Erichson, 1847) & 0 & 0 & 0 & 0 & 0 & 5 & 0 & 0 \\
\hline Deltochilum gibbosum (Fabricius, 1775) & 0 & 0 & 0 & 2 & 0 & 0 & 0 & 0 \\
\hline Deltochilum amazonicum, Bates 1887 & 127 & 3 & 0 & 0 & 0 & 7 & 225 & 0 \\
\hline Deltochilum septemstriatum Paulian, 1938 & 0 & 0 & 0 & 0 & 0 & 0 & 8 & 0 \\
\hline Deltochilum sp. [085] & 0 & 0 & 0 & 0 & 0 & 7 & 0 & 0 \\
\hline Deltochilum sp. [086] & 0 & 0 & 0 & 0 & 0 & 0 & 0 & 44 \\
\hline Diabroctis cadmus Harold, 1868 & 4 & 0 & 0 & 0 & 0 & 0 & 0 & 0 \\
\hline Dichotomius agenor Harold, 1869 & 952 & 68 & 327 & 127 & 0 & 5 & 58 & 3 \\
\hline Dichotomius bitiensis (Gillet, 1911) & 0 & 0 & 0 & 0 & 0 & 0 & 2 & 0 \\
\hline Dichotomius boreus Olivier, 1789 & 0 & 0 & 0 & 0 & 0 & 0 & 214 & 0 \\
\hline Dichotomius coenosus Erichson, 1848 & 12 & 0 & 3 & 2 & 0 & 0 & 0 & 0 \\
\hline Dichotomius inachus (Erichson, 1847) & 5 & 1 & 0 & 0 & 0 & 0 & 0 & 0 \\
\hline Dichotomius lucasi Harold, 1869 & 0 & 0 & 0 & 0 & 0 & 0 & 9 & 0 \\
\hline Dichotomius nisus (Oliver, 1789) & 0 & 27 & 0 & 0 & 0 & 0 & 0 & 0 \\
\hline Dichotomius protectus Harold, 1867 & 0 & 0 & 0 & 0 & 39 & 0 & 0 & 558 \\
\hline Dichotomius sp. [A16] & 0 & 0 & 0 & 534 & 0 & 0 & 0 & 0 \\
\hline Dichotomius validipilosus (Luederwaldt, 1931) & 0 & 0 & 0 & 0 & 4 & 0 & 0 & 0 \\
\hline Digitonthophagus gazella (Fabricius, 1787) & 130 & 1 & 0 & 0 & 0 & 0 & 0 & 0 \\
\hline Eurysternus balachowskyi Halffter y Halffter, 1977 & 0 & 0 & 0 & 0 & 0 & 0 & 4 & 0 \\
\hline Eurysternus caribaeus (Herbst, 1789) & 0 & 0 & 0 & 0 & 0 & 6 & 253 & 31 \\
\hline Eurysternus hamaticollis Balthasar, 1939 & 0 & 0 & 0 & 0 & 0 & 0 & 8 & 0 \\
\hline Eurysternus impressicollis Castelnau, 1840 & 139 & 0 & 0 & 0 & 0 & 0 & 0 & 0 \\
\hline Eurysternus marmoreus Castelnau, 1840 & 0 & 0 & 0 & 0 & 25 & 0 & 0 & 0 \\
\hline Eurysternus mexicanus Harold, 1869 & 79 & 1 & 0 & 0 & 23 & 14 & 93 & 0 \\
\hline Eurysternus plebejus Harold, 1880 & 0 & 0 & 0 & 0 & 0 & 2 & 0 & 3 \\
\hline Eurysternus sp. [A17] & 0 & 0 & 0 & 1 & 0 & 0 & 0 & 0 \\
\hline Eurysternus superbus Génier, 2009 & 0 & 0 & 0 & 0 & 0 & 0 & 0 & 238 \\
\hline Eurysternus ventricosus Gill, 1990 & 0 & 0 & 0 & 0 & 0 & 0 & 1 & 0 \\
\hline Malagoniella astyanax (Blanchard, 1845) & 4 & 1 & 0 & 4 & 0 & 0 & 0 & 0 \\
\hline Ontherus appendiculatus Mannerheim, 1829 & 0 & 31 & 0 & 0 & 0 & 0 & 0 & 0 \\
\hline Ontherus gilli Génier, 1996 & 0 & 0 & 0 & 0 & 187 & 0 & 0 & 0 \\
\hline Ontherus sulcator Fabricius, 1775 & 0 & 0 & 0 & 0 & 0 & 0 & 31 & 0 \\
\hline Onthophagus acuminatus Harold, 1880 & 14 & 0 & 0 & 0 & 0 & 0 & 0 & 0 \\
\hline Onthophagus curvicornis Latreille, 1811 & 0 & 0 & 0 & 0 & 495 & 0 & 0 & 72 \\
\hline Onthophagus landolti (Harold, 1880) & 6 & 29 & 0 & 0 & 0 & 0 & 0 & 0 \\
\hline Onthophagus marginicollis Harold, 1880 & 1388 & 690 & 0 & 0 & 0 & 199 & 33 & 3 \\
\hline Onthophagus rhinophyllus Harold, 1868 & 0 & 0 & 0 & 0 & 0 & 327 & 27 & 281 \\
\hline Onthophagus rubrescens Blanchard, 1843 & 0 & 1 & 0 & 0 & 0 & 0 & 179 & 0 \\
\hline Onthophagus sp. [071] & 139 & 0 & 0 & 0 & 0 & 0 & 0 & 0 \\
\hline Onthophagus sp. [A18] & 0 & 0 & 0 & 25 & 0 & 0 & 0 & 0 \\
\hline Onthophagus sp. [A19] & 0 & 0 & 0 & 98 & 0 & 0 & 0 & 0 \\
\hline Onthophagus sp. [A20] & 0 & 0 & 0 & 1 & 0 & 0 & 0 & 0 \\
\hline Onthophagus sp. [A21] & 0 & 0 & 0 & 0 & 3 & 0 & 0 & 0 \\
\hline
\end{tabular}


ANEXO / APPENDIX (Continucación / Continued)

\begin{tabular}{|c|c|c|c|c|c|c|c|c|}
\hline Especie/morfoespecie & NM05 & NM08 & NM09 & NM24 & NM26 & NM92 & NM93 & NM97 \\
\hline Onthophagus sp. [A22] & 0 & 0 & 50 & 0 & 0 & 0 & 0 & 0 \\
\hline Onthophagus sp. [A23] & 0 & 0 & 71 & 0 & 0 & 0 & 0 & 0 \\
\hline Oxysternon festivum Linnaeus, 1767 & 0 & 0 & 0 & 9 & 0 & 0 & 18 & 0 \\
\hline Phanaeus hermes Harold, 1868 & 15 & 0 & 0 & 0 & 0 & 0 & 0 & 0 \\
\hline Phanaeus prasinus Harold, 1868 & 0 & 5 & 1 & 12 & 39 & 66 & 14 & 0 \\
\hline Pseudocanthon sp. [060] & 112 & 14 & 0 & 0 & 0 & 118 & 0 & 0 \\
\hline Pseudocanthon sp. [A24] & 0 & 0 & 0 & 1037 & 0 & 0 & 0 & 0 \\
\hline Pseudocanthon sp. [A25] & 0 & 0 & 3 & 0 & 0 & 0 & 0 & 0 \\
\hline Pseudocanthon xanthurus Blanchard, 1843 & 12 & 37 & 0 & 0 & 0 & 0 & 1 & 0 \\
\hline Scatimus fernandezi Martínez, 1988 & 0 & 0 & 0 & 0 & 0 & 2 & 0 & 0 \\
\hline Scybalocanthon cyanocephalus Harold, 1868 & 0 & 0 & 0 & 0 & 0 & 0 & 14 & 0 \\
\hline Scybalocanthon sp. [A26] & 0 & 0 & 0 & 5 & 0 & 0 & 0 & 0 \\
\hline Sylvicanthon bridarollii Martínez, 1949 & 0 & 0 & 0 & 0 & 0 & 0 & 227 & 0 \\
\hline Uroxys sp. [029] & 0 & 0 & 0 & 0 & 0 & 0 & 10 & 0 \\
\hline Uroxys sp. [045] & 30 & 0 & 0 & 0 & 0 & 1 & 0 & 0 \\
\hline Uroxys sp. [081] & 0 & 0 & 0 & 0 & 0 & 5 & 0 & 0 \\
\hline Uroxys sp. [091] & 0 & 0 & 0 & 0 & 0 & 0 & 0 & 103 \\
\hline Uroxys sp. [093] & 0 & 0 & 0 & 0 & 0 & 0 & 0 & 220 \\
\hline Uroxys sp. [094] & 0 & 0 & 0 & 0 & 0 & 0 & 0 & 120 \\
\hline Uroxys sp. [A27] & 0 & 0 & 0 & 0 & 59 & 0 & 0 & 0 \\
\hline Uroxys sp. [A28] & 0 & 0 & 0 & 0 & 190 & 0 & 0 & 0 \\
\hline Uroxys sp. [A29] & 0 & 0 & 0 & 0 & 105 & 0 & 0 & 0 \\
\hline Uroxys sp. [A30] & 0 & 0 & 0 & 0 & 61 & 0 & 0 & 0 \\
\hline Uroxys sp. [A31] & 0 & 0 & 0 & 14 & 0 & 0 & 0 & 0 \\
\hline Uroxys sp. [A32] & 0 & 0 & 14 & 0 & 0 & 0 & 0 & 0 \\
\hline Total & 10860 & 3206 & 2723 & 4484 & 2767 & 3920 & 5390 & 6987 \\
\hline
\end{tabular}

